\title{
A Wide-stopband Low-pass Filter Design Based on Multi-period Taper-etched EBG Structure
}

\author{
Yin-Chi Chen, An-Shyi Liu, and Ruey-Beei Wu \\ Department of Electrical Engineering and Graduate Institute of Communication Engineering National \\ Taiwan University Taipei, Taiwan 106, R.O.C.
}

\begin{abstract}
Electromagnetic bandgap (EBG) microstrip structures have been proposed in the application of antenna and microwave filter design. The paper demonstrates a design procedure for the EBG structure to achieve a wide stopband lowpass filter, by combining tapered etched holes and multi-period EBG structure. The measurement result shows the rejection band is from $5.2 \mathrm{GHz}$ to $25 \mathrm{GHz}$, which meets the simulation result.
\end{abstract}

Index Terms - Electromagnetic bandgap, multi-period EBG

\section{INTRODUCTION}

A periodic structure which may exhibit the rejection of a certain frequency band is called photonic bandgap (PBG) or electromagnetic bandgap (EBG) structure. The applications of EBG structure in microstrip technology investigated in literatures are concentrated on antenna and microwave filter design [1]-[7]. The main mechanism of EBG integrated antenna is to suppress surface wave while the EBG integrated filter is to reject a frequency band. The aim of this work is to design an EBG structure as a wide stopband low-pass filter.

In conventional microwave filter design, the undesired frequency suppression has been usually implemented using shorted-circuited stub or surface-mounted capacitor. The shorted-circuited stub is one-half guided wavelength long at the frequency to be rejected and occupies some circuit area. On the other hand, the surface-mounted capacitor is suitable for low-frequency applications and its parasitic effect becomes severe as the frequency increases. It is difficult to estimate the parasitic effect owing to fabrication discrepancy in the design procedure. Therefore, another resolution which is a simple pattern of periodic holes or structures etched in ground plane beneath the strip conductor, has been proposed for low-pass filter design [2]-[7].

Two-dimensional (2-D) periodic patterns etched in ground plane for microstrip lines were first proposed [2]-[3]. Owing to the highly confined field around the strip conductor, the structure is then reduced to one-dimensional (1-D) ones in some literature [4]-[7]. Although a theoretic study to analyze the rejection frequency band of 1-D EBG microstrip structure has been proposed [4], it considers only the design that the ratio of radius of etched circular holes to separation between

This work was supported in part by the National Science Council, Taiwan, ROC, under Grant NSC 93-2752- E-002-003-PAE.

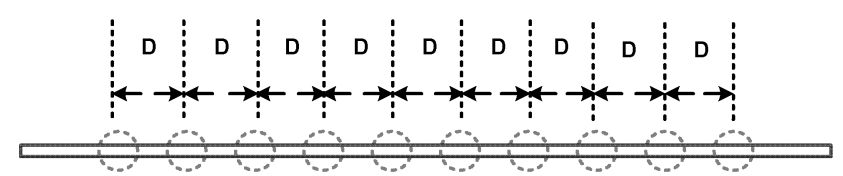

(a)

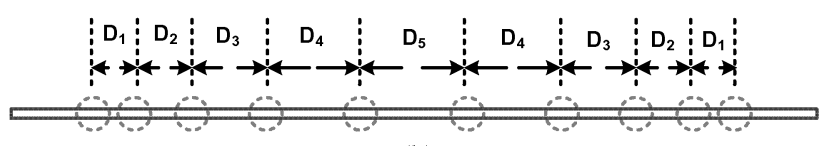

(b)

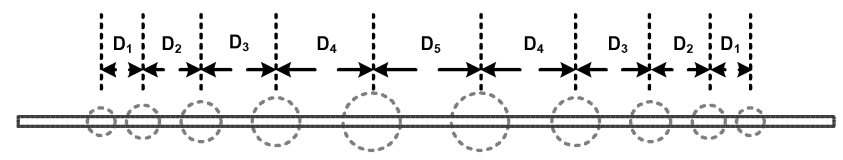

Fig. 1 Schematic representations of different EBG structures. (a) single-period, (b) multi-period, and (c) multi-period with tapered etched holes.

centers of adjacent holes are kept the same. Such a design does not provide optimal bandwidth of rejection band for a given number of etched holes. Other designs for wider rejection band can be implemented, such as by cascaded EBG microstrip circuit [3], taper structure in ground plane [6], or multi-period EBG structure [6]-[7].

The structure proposed in this paper is based on a combination of tapered etched holes and multi-period EBG structure. With suitable choice of the radius ratio and the separation of adjacent holes, a novel wide rejection band, which is from $5.2 \mathrm{GHz}$ to $25 \mathrm{GHz}$, can be obtained. The first section describes how the EBG structure was designed. It is followed by a section describing the simulation and measured results, and then is the conclusion.

\section{DESIGN AND ANALYSIS OF EBG STRUCTURES}

Several typical EBG structures with etched circular holes in ground plane of a $50 \Omega$ microstrip line are shown in Fig. 1. In the design procedure, single-period structures shown in Fig. 1(a) were first analyzed by Ansoft Ensemble 8.0 [8] with separations $\mathrm{D}$ as a parameter. The central frequency, depth, and bandwidth of the forbidden band for the 1-D EBG structure can be determined by the separation of adjacent 
holes in the ground plane $(D)$, the hole radius $(R)$, and the number of holes $(N)$ [4].

For a single-period EBG structure, the central frequency of the first stopband can be roughly determined by the period $D$ using the formula [1]:

$$
f=\frac{c}{\sqrt{\varepsilon_{e f f}}} \cdot \frac{1}{\lambda_{g}}
$$

where $c$ is the speed of light in free space, and $\lambda_{g}=2 \cdot D$ is the Bragg condition. Recently, a more accurate expression for the Bragg condition has been proposed [5], which takes into account the change in the effective dielectric constant of the microstrip line $\varepsilon_{\text {eff }}$ produced by the pattern etched in the ground plane. For a microstrip line with etched ground plane, the effective dielectric constant $\varepsilon_{\text {eff }}$ can be estimated as that of the line with unperturbed ground plane, i.e.,

$$
\varepsilon_{\text {eff }}=\frac{\varepsilon_{r}+1}{2}+\frac{\varepsilon_{r}-1}{2} \cdot \frac{1}{\sqrt{1+12 d / W}}
$$

where $d$ and $W$ are the thickness of the substrate and the width of the line, respectively.

The structure considered here has parameters $\varepsilon_{r}=3.38$ (Rogers RO4003), $d=20 \mathrm{mils}$, and $W=1.2 \mathrm{~mm}$, corresponding to a $50 \Omega$ conventional microstrip line. To begin with, the unit lattice is chosen to be a circle with uniform radius for investigating the bandgap characteristics. The bandwidth of bandgap can be estimated by full-wave analysis with the separation of aduacent holes, $D$, as a parameter. In order to achieve at non-overlapping band gap, one can then determine a set of separations between adjacent circles, $D_{i}$ 's. The radius of etched circles, $R$, is chosen between the line width and one half-wavelength. A small circle degrades the structure into a conventional microstrip and the band gap vanishes. A large one acts as a slot patch when the radius reaches to one halfwavelength and increases radiation loss at high frequency.

The second step is to determine the taper ratio of adjacent circles. For convenience, the ratio $R_{i+1} / R_{i}$ is set to be a constant so as to confine the radii of etched circles within the constraint mentioned in the above paragraph. With a suitable selection of the taper ratio, a wide-band rejection band can be obtained.

\section{SimulATION AND MEASUREMENT}

In order to investigate the stopband behavior of the proposed EBG structure, five single-period structures are analyzed, as shown in Fig. $1(a)$. The period $D=5 \mathrm{~mm}, 7 \mathrm{~mm}, 9 \mathrm{~mm}, 11.5 \mathrm{~mm}$, and $13 \mathrm{~mm}$ is a parameter, while the etched holes radius is fixed at $R=2 \mathrm{~mm}$. Simulation results of transmission $\left(\mathrm{S}_{21}\right)$ are shown in Fig.2. The central frequencies of the first stopband are $18.5 \mathrm{GHz}, 13.2 \mathrm{GHz}, 10.3 \mathrm{GHz}, 8.1 \mathrm{GHz}$, and $7.1 \mathrm{GHz}$ respectively, which shows that the central frequency of the stopband obtained by using (1) is a good estimation.

Also noticed in Fig.2 is the non-overlapping stopband. This indicates that a linear combination of these periods may contribute to a wider stopband response. In addition, the ratio $R / D$ 's are $0.4,0.29,0.22,0.17$, and 0.15 respectively. As shown in Fig.2, a larger $R / D$ results in a wider stopband bandwidth. It is in good agreement with the result shown in [1].

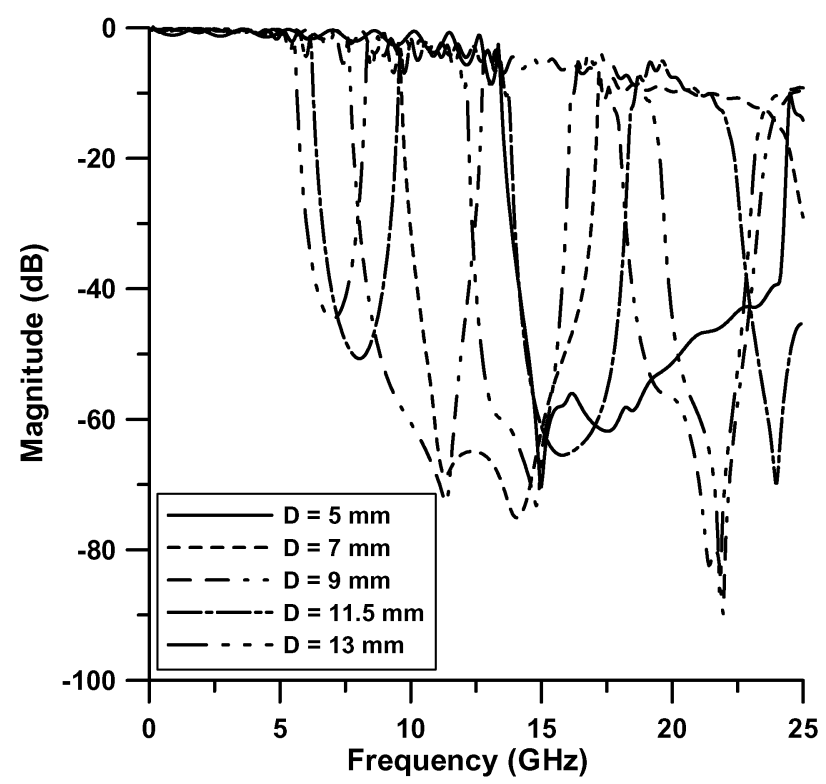

Fig. 2 Simulated $\mathrm{S}_{21}$ parameter of single period 1-D EBG microstrip structure.

\begin{tabular}{cccc}
\multicolumn{5}{c}{ TABLE I } \\
DIMENSIONS FOR MULTIPERIOD \\
EBG STRUCTURE \\
\hline i & $\mathbf{D}_{\mathbf{i}}(\mathrm{mm})$ & $\mathbf{R}_{\mathrm{i}}(\mathrm{mm})$ & $\mathbf{R}_{\mathrm{i}+1} / \mathbf{R}_{\mathrm{i}}$ \\
\hline 1 & 5 & 2 & \\
2 & 7 & 2 & 1.0 \\
3 & 9 & 2 & 1.0 \\
4 & 11.5 & 2 & 1.0 \\
5 & 13 & 2 & 1.0 \\
\hline
\end{tabular}

The five single-period EBG structures are cascaded in series to form a 1-D multi-period EBG structure, as shown in Fig.1(b). Table I lists the separations $D_{i}$ of adjacent circles. The simulated S parameters are shown in Fig.3. It is found that the stopband of this multi-period EBG structure is widened owing to the cascading of the individual stopbands.. The stopband obtained for the multi-period EBG is just like a combination of the stopbands of the five single-period EBG. The presence of a passband around $10 \mathrm{GHz}$ in Fig.3 depicts that the design is not good enough. A better approach for performance upgrade is to adopt a tapered EBG microstrip device [4], with the parameters listed in Table II. The simulated results in Fig. 4 show that the passband around 10 $\mathrm{GHz}$ is successfully suppressed, and an impressive wide 
stopband and high rejection level can be observed. Good agreement is also found between the measurements and the full-wave simulated data.

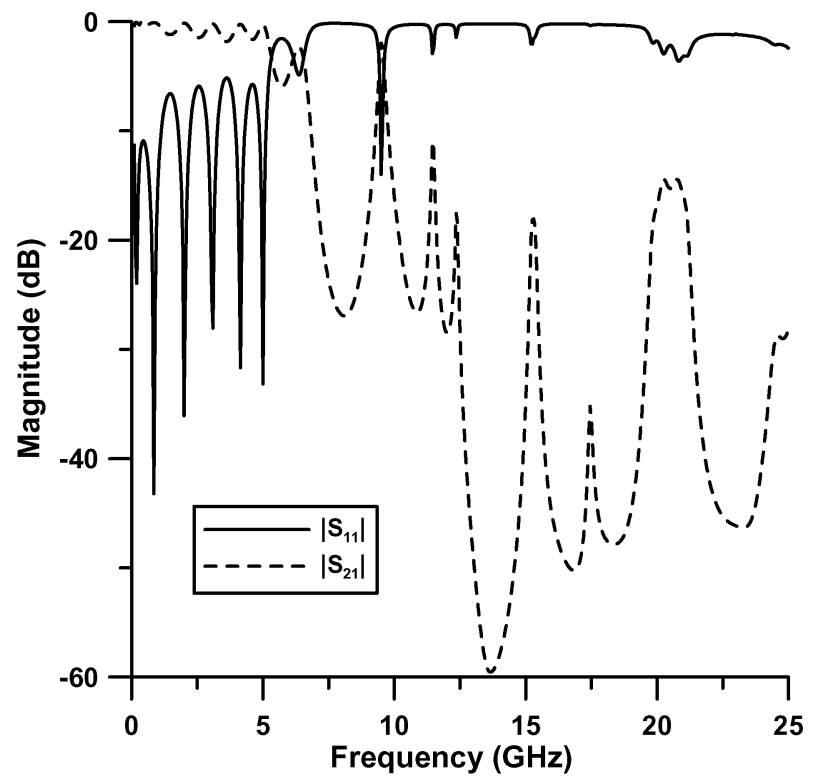

Fig. 3 Simulated $S_{21}$ parameter of multi-period 1-D EBG microstrip structure.

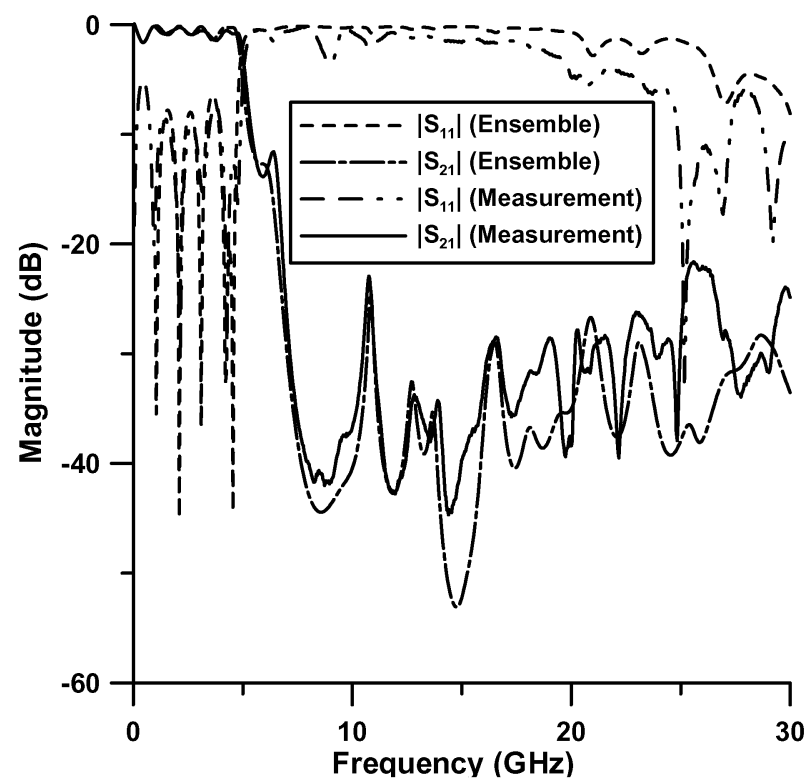

Fig. 4 Simulated and measured $\mathrm{S}_{21}$ parameter of optimal multiperiod 1-D EBG microstrip structure.
TABLE II OPTIMAL DIMENSIONS FOR MULTIPERIOD EBG STRUCTURE

\begin{tabular}{cccc}
\hline $\mathbf{i}$ & $\mathrm{D}_{\mathbf{i}}(\mathrm{mm})$ & $\mathrm{R}_{\mathrm{i}}(\mathrm{mm})$ & $\mathbf{R}_{\mathrm{i}+1} / \mathbf{R}_{\mathbf{i}}$ \\
\hline 1 & 5 & 1.67 & 1.2 \\
2 & 7 & 2.00 & 1.2 \\
3 & 9 & 2.40 & 1.2 \\
4 & 11.5 & 2.88 & 1.2 \\
5 & 13 & 3.46 & \\
\hline
\end{tabular}

\section{CONCLUSION}

The behavior of EBG structures with etched circles in ground plane is studied. It is shown that their associated stopbands can be joined together to form a very wide stopband by cascading different periods. The multi-period EBG structure constructed according to this principle still remains in 1-D, which is of immediate interest for the design of filters.

It is also demonstrated that a wide stopband low pass filter can be achieved by employing the tapered etched circles in ground plane. This novel device can be used to perform broadband filtering of undesired frequencies, overcoming the narrowband techniques commonly used today. Other possible applications, such as filters, frequency-selective surfaces, and efficient antenna structures, require further studies.

\section{REFERENCES}

[1] R. Coccioli, F. R. Yang, K. P. Ma, and T. Itoh, "Aperturecoupled patch antenna on UC-PBG substrate," IEEE Trans. Microwave Theory Tech., vol. 47, pp. 2123-2130, Nov. 1999.

[2] V. Radisic, Y. Qian, R. Coccioli, and T. Itoh, "Novel 2-D photonic bandgap structure for microstrip lines", IEEE Microwave Guided. Wave Lett., vol. 8, pp. 69-71, Feb. 1998.

[3] I. Rumsey, M. Piket-May, and P.K Kelly, "Photonic bandgap structures used as filters in microstrip circuits", IEEE Microwave Guided Wave Lett., vol. 8, pp. 336-338, Oct. 1998.

[4] M.A.G. Laso, M.J. Erro, D. Benito, M.J. Garde, T. Lopetegi, F. Falcone, and M. Sorolla, "Analysis and design of 1-D photonic bandgap microstrip structures using a fiber grating model', Microwave Opt Technol Lett., vol. 22, pp. 223-226, Aug. ,1999.

[5] M.A.G. Laso, T. Lopetegi, M.J. Erro, D. Benito, M.J. Garde, and M, Sorolla, "Novel wideband photonic bandgap microstrip structures", Microwave Opt. Technol. Lett., vol. 5, pp. 357-360, 2000.

[6] M. A. G. Laso, T. Lopetegi, M. J. Erro, D. Benito, M. J. Garde, and M. Sorolla, "Multiple-frequency-tuned photonic bandgap microstrip structures," IEEE Microave Guided Wave Lett., vol. 10, pp. 220-222, Jun. 2000.

[7] C.C. Chiau, X. Chen and C. Parin,"Multiperiod EBG structure for wide stopband circuits", IEE Proc.Microwave Antennas Propag., pp. 489- 492, Vol.150, Dec. 2003.

[8] Ansoft Ensemble, software package developed by Ansoft Co. 